ISSN 0103-5150

Fisioter. Mov., Curitiba, v. 24, n. 3, p. 503-511, jul./set. 2011 Licenciado sob uma Licença Creative Commons

\title{
Alterações neuromusculares no quadril associadas a entorses do tornozelo: revisão de literatura
}

\author{
Neuromuscular alterations on the hip associated to ankle sprains: \\ literature review
}

\section{Rodrigo Scattone da Silva ${ }^{[a]}$, Aline Pereira da Silva ${ }^{[b]}$, Denise Aparecida Sônego ${ }^{[c]}$, Nilma Marques de Paula ${ }^{[\mathrm{d}]}$}

[a] Aluno bolsista da Secretaria de Estado da Saúde, curso de Aprimoramento Profissional em Fisioterapia em Ortopedia e Traumatologia da Universidade Estadual de Campinas (Unicamp), Campinas, SP - Brasil, email: rodrigo_scattone@hotmail.com

[b] Aluna bolsista da Secretaria de Estado da Saúde, curso de Aprimoramento Profissional em Fisioterapia em Ortopedia e Traumatologia da Universidade Estadual de Campinas (Unicamp), Campinas, SP - Brasil, email: alineufscar@gmail.com

[c] Supervisora do curso de Aprimoramento Profissional em Fisioterapia em Ortopedia e Traumatologia da Universidade Estadual de Campinas (Unicamp), Campinas, SP - Brasil, email: sonego@hc.unicamp.br

[d] Supervisora do curso de Aprimoramento Profissional em Fisioterapia em Ortopedia e Traumatologia da Universidade Estadual de Campinas (Unicamp), Campinas, SP - Brasil, email: nilma@hc.unicamp.br

\section{Resumo}

Introdução: 0 tornozelo é a articulação mais frequentemente lesada na vida diária e nas atividades esportivas, sendo a entorse em inversão a lesão mais comum, com importantes repercussões funcionais e alto índice de recorrência após um evento de entorse primário. Os efeitos da lesão no tornozelo não se restringem a essa articulação, de modo que alterações na ativação e força da musculatura do quadril têm sido observadas após entorses em inversão do tornozelo. Essas alterações podem modificar o posicionamento do pé no instante do toque do calcanhar no solo, tornando o tornozelo mais suscetível à lesão por inversão. Objetivos: 0 propósito deste estudo foi realizar uma revisão dos estudos da literatura que analisaram alterações neuromusculares presentes na articulação do quadril associadas a entorses em inversão do tornozelo. Materiais e métodos: Foi realizada uma busca em bases eletrônicas (PubMed, MEDLINE, Cochrane, Web of Science, PEDro, SciELO, LILACS e Embase), do ano de 1966 até 2009, tendo sido encontrados 13 trabalhos considerados pertinentes ao tema. Resultados: Desses estudos, nove observaram alterações de 
força e/ou recrutamento dos músculos do quadril após entorses do tornozelo, sugerindo-se que as consequências da lesão local manifestam-se também em estruturas proximais no membro inferior, com possível influência na ocorrência de recidivas. Apenas dois trabalhos foram análises prospectivas e não evidenciaram alterações neuromusculares no quadril antes da lesão do tornozelo, sugerindo-se que as alterações de força e/ou recrutamento dos músculos do quadril são provavelmente secundárias à lesão articular distal. Conclusão: Mais estudos, com maior uniformidade metodológica, são necessários para maior elucidação sobre a questão.

Palavras-chave: Músculos do quadril. Entorse em inversão. Tornozelo. Eletromiografia.

\section{Abstract}

Introduction: The ankle is the most frequently injured joint both in athletics and daily life, and the inversion sprain is the most common injury with significant functional repercussion, given the high recurrence rate after a primary sprain. The effects of ankle injury are not restricted to that joint and changes in the recruitment and strength of the hip muscles have been found after ankle inversion sprains. These changes may implicate in modified positions of the foot in gait during heel strike, making the ankle more susceptible to inversion injury. objectives: The purpose of this article was to perform a review of the literature studies that have analyzed the neuromuscular alterations of the hip joint associated with ankle inversion sprains. Materials and methods: A search in electronic databases (PubMed, MEDLINE, Cochrane, Web of Science, PEDro, SciELO, LILACS and Embase) was performed from 1966 to 2009, with 13 studies found to be pertinent to this matter. Results: Among these, nine have found alterations in strength and/or recruitment of the hip muscles after an ankle sprain, suggesting that the implications of the local injury also manifest in proximal structures of the lower limb, with possible influence in recurrence of the injury. Only two articles were prospective analysis, and in those no evidence of neuromuscular alterations on the hip was found prior to the ankle injury, suggesting that the changes in strength and firing of the hip muscles are probably secondary to the distal lesion. Conclusion: More studies, with more methodological uniformity, are necessary for further elucidation on this matter.

Keywords: Hip muscles. Inversion sprain. Ankle. Electromyography.

\section{Introdução}

O tornozelo é a articulação mais frequentemente lesada na vida diária e nas atividades esportivas (1). A entorse do tornozelo é a lesão mais comumente observada (2), sendo o mecanismo por inversão responsável por $70 \%$ a $85 \%$ dos casos (3). A lesão aguda em inversão resulta em dano às estruturas laterais do tornozelo, que incluem os ligamentos laterais, as cápsulas das articulações subtalar e talocrural (4) e o nervo fibular superficial $(5,6)$. Tais alterações apresentam implicações clínicas importantes, uma vez que se observa instabilidade funcional (sensação de falseio e recorrência da lesão) em $15 \%$ a $60 \%$ dos casos após um evento de entorse primário (7).

Acredita-se que a lesão nas estruturas relacionadas ao sistema sensório-motor presentes no tornozelo seja uma das principais causas de recorrência das lesões em inversão $(8,9)$. Alguns autores têm observado alterações no tempo de resposta eletromiográfica dos músculos eversores do tornozelo em indivíduos portadores de instabilidade crônica do tornozelo (10-12). Contudo, outros autores não observaram tais diferenças em estudos similares (13-15), sugerindo que o tempo de resposta dessa musculatura à perturbação seria longo demais para proteger o tornozelo de uma inversão abrupta, de modo que outros fatores poderiam ser responsáveis pela estabilidade do membro inferior (6).

Os efeitos da lesão no tornozelo não se restringem ao tornozelo em si (1). Alterações de controle postural $(16,17)$ e na ativação e força da musculatura do quadril $(1,18-25)$ têm sido observadas após entorses em inversão do tornozelo, sugerindo-se que as consequências da lesão local manifestam-se também em estruturas proximais no membro inferior (1). A estabilidade do quadril e a força dos músculos dessa articulação são importantes para um 
melhor posicionamento do pé na fase de toque do calcanhar e para a mecânica adequada da marcha (21). Alguns autores têm ressaltado o controle adequado do quadril como fator essencial para a manutenção do controle da articulação do tornozelo e da estabilidade do membro inferior no plano frontal $(1,26,27)$. Alterações nos momentos abdutor e adutor do quadril, na fase de balanço da marcha, podem alterar o posicionamento do pé no instante do toque do calcanhar no solo (26), tornando o tornozelo mais suscetível a uma lesão por inversão (21).

Considerando-se a intricada relação cinemática que existe entre as articulações do membro inferior, torna-se necessária a investigação das disfunções presentes não só no foco, mas em regiões distantes da lesão $(1,21)$. Apesar das indicações de que a lesão por entorse em inversão do tornozelo possa estar relacionada com alterações neuromusculares na articulação do quadril, pouca atenção tem sido voltada para melhor elucidar essa questão, e os resultados dos estudos sobre o tema são contraditórios e pouco conclusivos. Assim, o propósito deste estudo foi realizar uma revisão dos estudos da literatura que realizaram uma avaliação das alterações neuromusculares presentes na articulação do quadril associadas à lesão por entorse em inversão do tornozelo.

\section{Metodologia}

Foi realizada uma busca eletrônica nas bases de dados PubMed, MEDLINE, Cochrane, Web of Science, PEDro, SciELO, LILACS e Embase, de 1966 até 2009, no período compreendido entre os dias 17 de agosto a 11 de setembro de 2009, cruzando-se as seguintes palavras-chave: ankle (tornozelo), sprain (entorse), hip (quadril) e instability (instabilidade). Foram incluídos no presente estudo os artigos que apresentaram os seguintes critérios: avaliação da força e/ou ativação muscular na articulação do quadril associada a lesões por entorse em inversão do tornozelo; e disponibilidade do artigo na íntegra nos idiomas inglês ou português. Foram excluídos do estudo os trabalhos que analisavam somente o desvio postural sem avaliar força e/ou ativação neuromuscular.

Os títulos e resumos dos trabalhos foram analisados e os estudos que preencheram os critérios de inclusão foram obtidos na íntegra. Cada artigo foi analisado pelos autores separadamente e, posteriormente, as informações coletadas pelos autores foram confrontadas e os dados que divergiram entre os autores foram analisados até que se chegasse a um consenso. 0 conteúdo dos artigos pesquisados deveria conter informações relevantes ao tópico explanado e ser passível de comparação com os demais artigos para que tal estudo fosse incluído na presente revisão.

\section{Resultados}

Nas bases de dados SciELO, LILACS e PEDro não foram encontrados estudos sobre o presente tema, tendo sido encontrados, nas demais bases de dados, 13 estudos pertinentes ao tema abordado, todos na língua inglesa. Dos trabalhos selecionados, um foi relato de caso (23), um foi revisão de literatura (28) e os 11 demais foram estudos laboratoriais $(1,18$ $22,24,25,29-31)$. Com relação às avaliações, cinco dos trabalhos analisaram a força muscular $(18,21$, 29-31), 7 consideraram o recrutamento muscular por eletromiografia $(1,19,20,22-25)$ e nenhum realizou avaliação de ambas as variáveis. Dois dos artigos foram análises prospectivas $(23,29)$ e 10 foram análises retrospectivas $(1,18-22,24,25,30,31)$.

Com relação ao grupo controle utilizado em cada trabalho, o membro inferior contralateral foi utilizado como controle em três estudos $(18,21,30)$; indivíduos sadios (sem história prévia de lesão nos membros inferiores) foram utilizados como controle em um estudo (25); e em quatro estudos $(1,19,20$, 29) foram utilizados, como controle, tanto o membro contralateral quanto indivíduos sadios para a realização de comparações. As principais características dos estudos estão expostas na Tabela 1.

\section{Discussão}

Lesões em inversão do tornozelo representam um alto percentual das visitas a centros de reabilitação, e o alto índice de recorrência dessa lesão tem atraído, mais recentemente, a atenção da comunidade científica (21). Atenção especial tem sido voltada para os fatores que contribuem para a recidiva das lesões, porém, trabalhos enfocando as alterações neuromusculares da articulação do quadril ainda são escassos. Pressupostos teóricos têm sido levantados na tentativa de elucidar a questão, mas a escassez de evidências dificulta a comprovação da 
Tabela 1 - Caracterização dos artigos incluídos na revisão

(Continua)

\begin{tabular}{|c|c|}
\hline Autores & Caracterização da amostra \\
\hline $\begin{array}{l}\text { Nicholas et al., } \\
1976 \text { [18] }\end{array}$ & $\begin{array}{l}\text { Grupo acometido: } 11 \text { indivíduos (média } \\
\text { de idade: } 30 \text { anos) com entorse crôni- } \\
\text { ca de tornozelo e/ou estiramentos ou } \\
\text { anormalidades anatômicas do pé. } \\
\text { Grupo controle: membro contralateral } \\
\text { não envolvido. }\end{array}$ \\
\hline $\begin{array}{l}\text { Bullock-Saxton, } \\
1994 \text { [19] e } \\
\text { Bullock-Saxton } \\
\text { et al., 1994 [20] }\end{array}$ & $\begin{array}{l}\text { Grupo acometido: } 20 \text { indivíduos do gê- } \\
\text { nero masculino ( } 18-35 \text { anos de idade) } \\
\text { com história prévia de entorses de tor- } \\
\text { nozelo unilaterais severas (graus II e III). } \\
\text { Grupo controle: } 11 \text { indivíduos saudáveis } \\
\text { do gênero masculino. }\end{array}$ \\
\hline
\end{tabular}

Beckman e

Buchanan, 1995

[1]

Grupo hipermóvel: 10 indivíduos (idade média: $32,3 \pm 5,7$ ) com no mínimo $10^{\circ}$ de excesso de inversão do tornozelo e com pelo menos uma entorse em inversão do tornozelo nos últimos dois anos. Grupo controle (GC): 10 indivíduos saudáveis (idade média: 29,8 $\pm 3,6$ ).

Variáveis avaliadas

Avaliou-se a produção de torque dos músculos quadríceps, isquiotibiais, abdutores, adutores e flexores do quadril em dinamômetro isocinético em três diferentes velocidades $(5$, 15 e $30 \mathrm{rpm})$.

Avaliaram-se, por eletromiografia (EMG), os músculos extensores da coluna lombar (bilateralmente) e os músculos bíceps femoral e glúteo máximo ipsilaterais à lesão, durante a extensão do quadril em prono com velocidade controlada.

Avaliação por EMG do glúteo médio (bilateralmente) durante simulação de entorse em inversão em plataforma $\left(30^{\circ}\right.$ de inversão na velocidade de $700 \%$ seg).

Foram realizados 20 testes para cada membro inferior de maneira aleatória.
Resultados

Observou-se menor torque abdutor e adutor do quadril do lado acometido quando comparado com o membro contralateral na velocidade mais baixa.

Observou-se atraso na ativação do músculo glúteo máximo no teste em ambos os membros do grupo acometido com relação ao grupo controle. Não houve diferença entre os membros acometido e contralateral.

0 músculo glúteo médio de ambos os lados do grupo hipermóvel apresentou menor tempo de latência quando comparado com $0 \mathrm{GC}$.

Observou-se, também, que no grupo hipermóvel o glúteo médio contralateral ativou-se antes do ipsilateral, durante a inversão dos tornozelos.

Antes do contato do calcanhar com o solo, o reto femoral do grupo IF apresentou maior atividade quando comparado com 0 GC. Após o contato calcanhar, não houve diferença na atividade do reto femoral entre os grupos.

Os abdutores do quadril do lado acometido apresentaram menor força muscular quando comparados ao membro contralateral. Não houve diferença entre os grupos com relação à força dos extensores do quadril.

A ativação muscular do glúteo máximo na 8a semana pós-lesão ocorreu mais precocemente, se comparada com a ativação ocorrida antes da lesão.
Avaliação por EMG do glúteo máximo ipsilateral à lesão durante extensão da perna em prono em velocidade não controlada. Foram realizadas três avaliações em ocasiões diferentes (pré-lesão, duas e oito semanas pós-lesão).

Avaliou-se, dentre outras variáveis, a força muscular isométrica dos abdutores, adutores e flexores do quadril de ambos os membros por meio de um dinamômetro manual.
Não houve diferença significativa na força muscular dos abdutores, adutores e flexores do quadril entre os atletas que sofreram lesão e os que não sofreram lesão. 
Tabela 1 - Caracterização dos artigos incluídos na revisão

(Conclusão)

\begin{tabular}{|c|c|c|c|}
\hline Autores & Caracterização da amostra & Variáveis avaliadas & Resultados \\
\hline $\begin{array}{l}\text { Fu e Hui-Chan, } \\
2007 \text { [24] }\end{array}$ & $\begin{array}{l}\text { Grupo entorses múltiplas de tornozelo } \\
\text { bilaterais (BMAS): } 19 \text { jogadores de bas- } \\
\text { quetebol do sexo masculino (média de } \\
\text { idade: } 20,4 \pm 1,0 \text { ). } \\
\text { Grupo controle }(\mathrm{GC}) \text { ): } 20 \text { indivíduos sa- } \\
\text { dios jogadores de basquetebol do sexo } \\
\text { masculino (média de idade: } 20,8 \pm 1,6 \text { ). }\end{array}$ & $\begin{array}{l}\text { Avaliou-se a EMG dos músculos } \\
\text { tensor da fáscia lata, fibular longo, } \\
\text { tibial anterior e gastrocnêmio medial } \\
\text { durante teste de quedas de } 30 \mathrm{~cm} \\
\text { de altura em duas condições: queda } \\
\text { inesperada e queda autoiniciada. }\end{array}$ & $\begin{array}{l}\text { Durante a queda autoiniciada, } \\
\text { houve atraso na ativação muscu- } \\
\text { lar do tensor da fáscia lata bilate- } \\
\text { ralmente no grupo BMAS quando } \\
\text { comparado ao GC. Contudo, ape- } \\
\text { nas no membro inferior esquerdo } \\
\text { esse atraso foi significativo. }\end{array}$ \\
\hline $\begin{array}{l}\text { Hubbard et al., } \\
2007 \text { [30] }\end{array}$ & $\begin{array}{l}30 \text { indivíduos (média de idade: } 20,3 \pm \\
1,3 \text { anos) que apresentavam instabili- } \\
\text { dade crônica unilateral de tornozelo e } \\
\text { membro contralateral sadio (sem his- } \\
\text { tória de fraturas, entorses ou cirurgias). }\end{array}$ & $\begin{array}{l}\text { Avaliou-se a força isométrica dos } \\
\text { abdutores e extensores do quadril, } \\
\text { por meio de dinamômetro manual. } \\
0 \text { trabalho buscou correlação entre } \\
\text { variáveis que determinam instabilida- } \\
\text { de mecânica e funcional do tornozelo. }\end{array}$ & $\begin{array}{l}\text { Não houve correlação de fraqueza } \\
\text { muscular dos abdutores e exten- } \\
\text { sores do quadril com as variá- } \\
\text { veis de instabilidade mecânica. } \\
\text { Houve correlação de fraqueza de } \\
\text { extensão e abdução do quadril e } \\
\text { pior desempenho no star balance } \\
\text { excursion test (tarefa para avalia- } \\
\text { ção de equilíbrio dinâmico). }\end{array}$ \\
\hline Niemuth, 2007 [28] & $\begin{array}{l}\text { Revisão de literatura relacionando fra- } \\
\text { queza dos músculos do quadril com } \\
\text { lesões nos membros inferiores. }\end{array}$ & $\begin{array}{l}\text { Ressaltam-se os trabalhos da litera- } \\
\text { tura que correlacionam fraqueza nos } \\
\text { abdutores e rotadores laterais do } \\
\text { quadril com lesões que incluem sín- } \\
\text { drome da dor patelofemoral, lesões } \\
\text { do ligamento cruzado anterior e en- } \\
\text { torses em inversão do tornozelo. }\end{array}$ & $\begin{array}{l}\text { Os trabalhos analisados parecem } \\
\text { indicar uma correlação significa- } \\
\text { tiva entre entorses em inversão } \\
\text { do tornozelo e alterações neuro- } \\
\text { musculares no quadril, envolven- } \\
\text { do redução de força e alteração } \\
\text { na ativação muscular. }\end{array}$ \\
\hline $\begin{array}{l}\text { Van Deun et al., } \\
2007 \text { [25] }\end{array}$ & $\begin{array}{l}\text { Grupo instabilidade crônica (CAI): } 10 \\
\text { indivíduos com instabilidade crônica de } \\
\text { tornozelo (média de idade: } 21 \text { anos). } \\
\text { Grupo controle (GC): } 30 \text { indivíduos sem } \\
\text { histórico de lesões nos membros infe- } \\
\text { riores (média de idade: } 22 \text { anos). }\end{array}$ & $\begin{array}{l}\text { Avaliação por EMG dos músculos } \\
\text { multífidos lombares, oblíquo inter- } \\
\text { no, reto abdominal, glúteo médio, } \\
\text { tensor da fáscia lata, vasto lateral, } \\
\text { vasto medial oblíquo, isquiotibiais } \\
\text { mediais, tibial anterior, fibular longo } \\
\text { e gastrocnêmio medial, na transi- } \\
\text { ção do apoio bipodal para unipodal. }\end{array}$ & $\begin{array}{l}\text { Todos os músculos avaliados, } \\
\text { com exceção do vasto lateral e do } \\
\text { vasto medial oblíquo, apresenta- } \\
\text { ram atraso na ativação muscular } \\
\text { no grupo CAl quando compara- } \\
\text { do com o GC, durante a realiza- } \\
\text { ção da tarefa. }\end{array}$ \\
\hline $\begin{array}{l}\text { Gribble e Robinson, } \\
2009 \text { [31] }\end{array}$ & $\begin{array}{l}\text { Grupo instabilidade crônica (CAI): } 15 \text { in- } \\
\text { divíduos com instabilidade crônica de } \\
\text { tornozelo (idade média: } 20,3 \pm 2,9 \text { anos). } \\
\text { Grupo controle (GC): } 15 \text { indivíduos sem } \\
\text { histórico de lesões nos membros infe- } \\
\text { riores (idade média: } 23,1 \pm 3,9 \text { anos). }\end{array}$ & $\begin{array}{l}\text { Avaliação isocinética concêntrica } \\
\text { dos flexores e extensores do qua- } \\
\text { dril, flexores e extensores do joelho } \\
\text { e dorsiflexores e flexores plantares } \\
\text { a } 60 \% \text { s. }\end{array}$ & $\begin{array}{l}\text { Observou-se menor força de fle- } \\
\text { xão e extensão do joelho no CAI } \\
\text { quando comparado ao GC. Não } \\
\text { se observou diferença significati- } \\
\text { va na força dos músculos flexo- } \\
\text { res e extensores do quadril entre } \\
\text { os grupos. }\end{array}$ \\
\hline
\end{tabular}

Fonte: Dados da pesquisa.

correlação entre controle neuromuscular pobre no quadril e entorses em inversão do tornozelo. Dentre as questões mais intrigantes está o estabelecimento de qual seria a causa e qual seria a consequência nesse contexto. Não se sabe ao certo se a fraqueza no quadril produziu alterações cinemáticas, que culminaram com a lesão distal, ou se as alterações secundárias à lesão do tornozelo produziram adaptações neuromusculares para manutenção do equilíbrio, manifestadas por meio de alterações de força e recrutamento dos músculos do quadril.

Tem sido cogitado que os momentos adutor e abdutor do quadril, gerados na fase de balanço, possam alterar o posicionamento do pé no solo na fase de toque de calcanhar da marcha (26). Sugere-se que, em indivíduos saudáveis, erros no posicionamento do pé no solo sejam corrigidos nas articulações subtalar e coxofemoral, trabalhando em sinergia, 
com erros menores sendo corrigidos pela musculatura do pé e erros maiores sendo corrigidos pelos músculos do quadril (26). As evidências parecem indicar que, em situações de lesão, surgem nesse sistema adaptações que desencadeiam alterações cinemáticas que podem predispor o indivíduo a recidivas da lesão primária.

Por perturbações externas do equilíbrio pode ocorrer um deslocamento lateral excessivo do centro de massa, e estratégias posturais de tornozelo são inicialmente utilizadas para conter os grandes desvios posturais laterais (21). Quando o tornozelo é incapaz de compensar adequadamente tais desvios, estratégias de quadril são iniciadas, auxiliando na prevenção de um momento inversor excessivo (32). Se o recrutamento e/ou a força dos músculos do quadril estiverem alterados, em virtude de uma lesão primária no tornozelo, a estabilidade postural ficará comprometida, especialmente no plano frontal, e o risco de recidivas, consequentemente, aumentará significativamente (21).

Nicholas et al. (18) foram os primeiros autores a evidenciar alterações de força nos músculos do quadril em indivíduos com lesões crônicas nos tornozelos e pés. Os autores observaram que indivíduos com tais lesões apresentavam menor pico de torque isocinético adutor e abdutor do quadril no membro inferior lesado quando comparado ao membro sem lesão (18). Desde então pouca atenção foi voltada para o assunto e somente mais recentemente as pesquisas têm voltado a enfocar as implicações das alterações neuromusculares proximais em lesões distais no membro inferior.

Bullock-Saxton (19) e Bullock-Saxton et al. (20) avaliaram os efeitos da lesão por entorse severa do tornozelo no padrão de recrutamento dos músculos do quadril no teste de extensão da perna em prono. Tal teste foi escolhido para esse propósito por avaliar estruturas distantes da região da lesão ao mesmo tempo em que simula um movimento funcional da marcha humana. Os autores evidenciaram retardo na ativação do glúteo máximo dos indivíduos com entorse severa no movimento de extensão do quadril quando comparados a um grupo controle. Curiosamente, o retardo de ativação do glúteo também foi observado no membro inferior contralateral sem lesão, o que indica a influência de mecanismos centrais na cadeia reflexa de eventos póslesão. Essas implicações distantes do foco de lesão, incluindo o membro inferior não lesado, indicam que a utilização do membro contralateral como grupo controle não seja a mais adequada nessas avaliações $(19,20)$. Corroborando com esses resultados, mais recentemente, Fu e Hui-Chan (24) observaram retardo de ativação do tensor da fáscia lata em 19 jogadores universitários de basquete, com múltiplas entorses bilaterais de tornozelo, em uma tarefa de aterrissagem de queda de altura, quando comparados com indivíduos sadios (24).

Van Deun et al. (25) avaliaram a atividade eletromiográfica de 14 músculos do tronco e do membro inferior na transição da posição de apoio bipodal para apoio unipodal em indivíduos com instabilidade crônica do tornozelo. A avaliação incluiu os músculos do quadril, dentre eles o glúteo médio e tensor da fáscia lata, homolaterais à lesão. Os autores observaram atraso na ativação de praticamente todos os músculos avaliados no grupo com instabilidade quando comparado com um grupo controle. Os autores sugerem que a instabilidade do tornozelo esteja associada a alterações na pré-ativação muscular ou feedforward, de modo que os indivíduos apresentam, após a lesão primária, maior dificuldade em lançar mão de estratégias compensatórias ao encontrarem-se em situações de maior demanda (25).

Algumas teorias têm sido propostas para justificar o retardo na ativação dos músculos do quadril após a lesão do tornozelo. Lesões capsulares e/ou ligamentares em animais desencadeiam alterações na atividade muscular não só dos músculos que cruzam a articulação, mas também de músculos em áreas distantes da lesão (33). Sugere-se que a interrupção de impulsos sensoriais que ocorre em virtude da lesão dos mecanorreceptores implique déficits locais de propriocepção e cinestesia. Desse modo, reflexos artrocinéticos alterados atuariam como fatores desencadeantes para reações adaptativas que culminariam com modificações significativas nos padrões de movimento de todo o membro (33). É também possível que a função dos músculos do quadril seja inibida após as entorses em inversão em razão da adoção de padrões alterados de marcha antálgica durante a fase sintomática da lesão. Padrões que envolvam minimização de contato do membro com o solo podem influenciar a musculatura do quadril, no sentido de redução de força e atraso de recrutamento (19).

Lehman (23) apresenta um relato de caso de avaliação prospectiva de um indivíduo do sexo feminino com o teste de extensão da perna em prono. A voluntária foi avaliada com o teste antes de sofrer uma 
entorse em inversão do tornozelo e, novamente, duas e oito semanas após a lesão. Contrariando os resultados de outros autores $(19,20)$, o glúteo máximo do membro lesado apresentou ativação precoce após oito semanas de lesão. Esses resultados indicam que as alterações neuromusculares do quadril observadas nos estudos retrospectivos são uma consequência da lesão das estruturas do tornozelo (23). 0 autor relata ainda inconsistência nos dados da avaliação com o teste da extensão da perna em prono, uma vez que foi observada grande variabilidade nos dados nas avaliações dentre diferentes sujeitos e entre múltiplas repetições de um mesmo sujeito $(34,35)$. Tal inconsistência pode ser relacionada ao fato de os autores não padronizarem a velocidade de realização do movimento de extensão do quadril como preconizado por outros autores $(19,20)$. Movimentos em diferentes velocidades podem produzir diferentes padrões de recrutamento, o que pode ter influenciado diretamente nos resultados.

Beckman e Buchanan (1) avaliaram indivíduos com hipermobilidade no tornozelo, realizando análise eletromiográfica do músculo glúteo médio bilateralmente, ao submeter os voluntários à inversão abrupta do tornozelo em uma plataforma rotatória que simulava o mecanismo de lesão. Contrariando os resultados de retardo de ativação dos músculos do quadril observados em outros trabalhos $(19,20$, $24,25)$, os autores observaram que os indivíduos hipermóveis apresentavam recrutamento precoce de ambos os glúteos médios, especialmente do glúteo contralateral, quando comparados a um grupo controle. Sugere-se que essa pré-ativação ocorra por alguns mecanismos. Uma hipótese é a de que os receptores articulares do tornozelo apresentariam efeito inibitório sobre a musculatura do quadril e, com o aumento da lassidão, tal mecanismo inibitório não se ativaria, de modo que o glúteo seria mais precocemente ativado em perturbações (1). É também possível que os indivíduos com hipermobilidade apresentem inconscientemente maior confiança em estratégias de quadril em virtude da lesão primária no tornozelo e, assim, ativem os estabilizadores do quadril precocemente em situações de instabilidade. Por fim, sugere-se que, por meio de mecanismos de feedforward, o sistema nervoso central aumentaria a atividade do motoneurônio gama para a musculatura do quadril, facilitando sua ativação para compensar a menor capacidade dos músculos do tornozelo em manter a estabilidade postural (1).
Delahunt et al. (22) apresentam achados que corroboram com essas hipóteses, uma vez que foi observado que indivíduos com instabilidade funcional do tornozelo apresentam maior atividade eletromiográfica do reto femoral imediatamente antes da fase de toque de calcanhar na marcha, indicando-se que esses indivíduos fazem maior uso de estratégias de quadril, na tentativa de compensar a instabilidade do tornozelo, quando comparados com indivíduos sadios (22). Acredita-se que o sistema nervoso central perceba o tornozelo pós-entorse como sendo incapaz de compensar pelo movimento de inversão em alta velocidade e que adaptações neuromusculares no quadril passem a ocorrer como mecanismos antecipatórios de compensação para manutenção da estabilidade (1).

Além de alterações de recrutamento dos músculos do quadril, alguns autores também têm evidenciado alterações na geração de força dessa musculatura (18, 21). Friel et al. (21) avaliaram a força isométrica abdutora e extensora do quadril de 23 indivíduos com instabilidade crônica do tornozelo. Os autores evidenciaram redução na força abdutora do quadril, no lado ipsilateral à lesão distal, quando comparado ao membro inferior contralateral, de modo que seus resultados corroboraram com os de Nicholas et al. (18). Os autores não observaram fraqueza dos músculos extensores do quadril pós-entorse, sugerindo que a força desse grupo muscular esteja menos afetada e menos envolvida nesse tipo de lesão (21). Como a lesão em inversão do tornozelo ocorre primariamente no plano frontal, é possível que os extensores do quadril, que atuam principalmente no plano sagital, apresentem um papel menos relevante para a estabilização do quadril que os músculos abdutores (21).

Contrariando esses resultados, McHugh et al. (29), em um estudo prospectivo com 169 atletas de colegial, não observaram correlação entre fraqueza dos músculos do quadril (flexores, adutores e abdutores) e maior índice de entorses em inversão do tornozelo em uma temporada. Hubbard et al. (30), ao avaliarem 30 indivíduos com instabilidade crônica de tornozelo, não observaram redução na força dos abdutores e extensores do quadril. Os autores observaram, contudo, correlação entre fraqueza nos músculos do quadril e desempenho pobre na tarefa de star balance excursion test, um teste utilizado para avaliação do equilíbrio dinâmico (30). Mais recentemente, Gribble e Robinson (31) compararam 15 indivíduos com instabilidade crônica do tornozelo com indivíduos sem lesão e também não 
evidenciaram redução na força extensora e flexora do quadril (31). Tais achados parecem indicar que as alterações, em termos de redução de força no quadril, não estão sempre presentes em indivíduos com instabilidade do tornozelo.

\section{Conclusões}

As evidências da literatura, apesar de escassas e contraditórias, indicam que os indivíduos que sofreram entorses em inversão do tornozelo tendem a apresentar alterações de força e/ou recrutamento dos músculos do quadril e que tais alterações são, provavelmente, secundárias à lesão articular distal. Apesar das indicações de que as alterações neuromusculares do quadril não constituem um fator causal para as lesões primárias em inversão do tornozelo, essas alterações implicam padrões cinemáticos aberrantes, com pobre controle do membro inferior no plano frontal, e provavelmente apresentam influência significativa na recorrência das lesões. Assim, tais alterações precisam ser avaliadas e consideradas no estabelecimento de intervenções terapêuticas, com implementação de condutas que visem ao ganho de força e à melhora no padrão de recrutamento da musculatura do quadril, corrigindo-se os padrões de movimento alterados para a prevenção de novas lesões.

\section{Referências}

1. Beckman SM, Buchanan TS. Ankle inversion injury and hypermobility: effect on hip and ankle muscle electromyography onset latency. Arch Phys Med Rehabil. 1995;76:1138-1143.

2. Derscheid GL, Brown WC. Rehabilitation of the ankle. Clin Sports Med. 1985;4(3):527-44.

3. Wolfe MW, Uhl TL, Mattacola CG, McCluskey LC. Management of ankle sprains. Am Fam Physician. 2001;63(1):93-104.

4. Holmer P, Sondergaard L, Konradsen L, Nielsen PT, Jorgensen LN. Epidemiology of sprains in the lateral ankle and foot. Foot Ankle Int. 1994;15(2):72-4.

5. Lemont H. Perineural fibrosis of the superficial peroneal nerve complicating ankle sprain: a case report. Foot Ankle. 1991;11(6):414.
6. Van Cingel RE, Kleinrensink G, Uitterlinden EJ, Rooijens PP, Mulder PG, Aufdemkampe G et al. Repeated ankle sprains and delayed neuromuscular response: acceleration time parameters. J Orthop Sports Phys Ther. 2006;36(2):72-9.

7. Kannus P, Renström P. Treatment for acute tears of the lateral ligaments of the ankle: operation, case, or early controlled motion. J Bone Joint Surg. 1991;73:305-12.

8. Konradsen L, Ravn JB. Ankle instability caused by prolonged peroneal reaction time. Acta Orthop Scand. 1990;61(5):388-90.

9. Jerosch J, Hoffstetter I, Bork H, Bischof M. The influence of orthoses on the proprioception of the ankle joint. Knee Surg Sports Traumatol Arthrosc. 1995;3(1):39-46.

10. Konradsen L, Ravn JB. Prolonged peroneal reaction time in ankle instability. Int J Sports Med. 1991;12(3): 290-2.

11. Brunt D, Andersen J, Huntsman B, Reinhert LB, Thorell AC, Sterling JC. Postural responses to lateral perturbation in healthy subjects and ankle sprain patients. Med Sci Sports Exerc. 1992;24(2):171-6.

12. Karlsson J, Andreasson GO. The effect of external ankle support in chronic lateral ankle joint instability: an electromyographic study. Am J Sports Med. 1992;20(3):257-61.

13. Johnson MB, Johnson CL. Electromyographic response of peroneal muscles in surgical and nonsurgical injured ankles during sudden inversion. J Orthop Sports Phys Ther. 1993;18(3):497-501.

14. Ebig M, Lephart SM, Burdett RG, Miller MC, Pincivero DM. The effect of sudden inversion stress on EMG activity of the peroneal and tibialis anterior muscles in the chronically unstable ankle. J Orthop Sports Phys Ther. 1997;26(2):73-7.

15. Fernandes N, Allison GT, Hopper D. Peroneal latency in normal and injured ankles at varying angles of perturbation. Clin Orthop Relat Res. 2000;(375):193-201.

16. Cornwall MW, Murrell P. Postural sway following inversion sprain of the ankle. J Am Podiatr Med Assoc. 1991;81(5):243-7.

17. Eechaute C, Vaes P, Duquet W. The dynamic postural control is impaired in patients with chronic ankle instability: reliability and validity of the multiple hop test. Clin J Sport Med. 2009;19(2):107-14. 
18. Nicholas JA, Strizak AM, Veras G. A study of thigh muscle weakness in different pathological states of the lower extremity. Am J Sports Med. 1976;4(6):241-8.

19. Bullock-Saxton J. Local sensation changes and altered hip muscle function following severe ankle sprain. Phys Ther. 1994;74(1):17-28.

20. Bullock-Saxton JE, Janda V, Bullock MI. The influence of ankle sprain injury on muscle activation during hip extension. Int J Sport Med. 1994;15(6):330-4.

21. Friel K, McLean N, Myers C, Caceres M. Ipsilateral hip abductor weakness after inversion ankle sprain. J Athl Train. 2006;41(1):74-9.

22. Delahunt E, Monaghan K, Caulfield B. Altered neuromuscular control and ankle joint kinematics during walking in subjects with functional instability of the ankle joint. Am J Sports Med. 2006;34(12): 1970-6.

23. Lehman GJ. Trunk and hip muscle recruitment patterns during the prone leg extension following a lateral ankle sprain: a prospective case study pre and post injury. Chiropr Osteopat. 2006;14:1-4.

24. Fu SN, Hui-Chan CWY. Modulation of pre-landing lower limb muscle responses in athletes with multiple ankle sprains. Med Sci Sport Exerc. 2007;39(10):1774-83.

25. Van Deun S, Staes FF, Stappaerts KH, Janssens L, Levin 0 , Peers K. Relationship of chronic ankle instability to muscle activation patterns during the transition from double-leg to single-leg stance. Am J Sports Med. 2007;35(2):274-81.

26. MacKinnon CD, Winter DA. Control of whole body balance in the frontal plane during human walking. J Biomech. 1993;26(6):633-44.

27. Sadeghi H, Sadeghi S, Prince F, Allard P, Labelle H, Vaughan CL. Functional roles of ankle and hip sagittal muscle moments in able-bodied gait. Clin Biomech. 2001;16(8):688-95.
28. Niemuth PE. The role of hip muscle weakness in lower extremity athletic injuries. Int Sport Med J. 2007;8(4): 179-92.

29. McHugh MP, Tyler TF, Tetro DT, Mullaney MJ, Nicholas SJ. Risk factors for noncontact ankle sprains in high school athletes: the role of hip strength and balance ability. Am J Sports Med. 2006;34(3):464-70.

30. Hubbard TJ, Kramer LC, Denegar CR, Hertel J. Correlations among multiple measures of functional and mechanical instability in subjects with chronic ankle instability. J Athl Train. 2007;42(3):361-6.

31. Gribble PA, Robinson RH. An examination of ankle, knee, and hip torque production in individuals with chronic ankle instability. J Strength Cond Res. 2009; 23(2):395-400.

32. Levangie PK. Gait. In: Norkin C, editor. Joint structure and function: a comprehensive analysis. Philadelphia: FA Davis; 1983. p. 435-82.

33. Wyke B. The neurology of joints. Ann R Coll Surg Engl. 1967;41(1):25-50.

34. Pierce MN, Lee WA. Muscle firing order during active prone hip extension. J Orthop Sport Phys Ther. 1990;12(1):2-9.

35. Lehman GJ, Lennon D, Tresidder B, Rayfield B, Poschar M. Muscle recruitment patterns during the prone leg extension. BMC Musculoskelet Disord. 2004;5:1-5.

Recebido: 08/12/2010

Received: $12 / 08 / 2010$

Aprovado: 25/03/2011

Approved: 03/25/2011 